\title{
Statistical mechanical theory of an oscillating isolated system: The relaxation to equilibrium
}

\author{
A. Pérez-Madrid ${ }^{\text {a) }}$ \\ Departament de Física Fonamental, Facultat de Física, Universitat de Barcelona, \\ Diagonal 647, 08028 Barcelona, Spain
}

(Received 28 March 2007; accepted 27 September 2007; published online 24 October 2007)

\begin{abstract}
In this Contribution we show that a suitably defined nonequilibrium entropy of an $\mathrm{N}$-body isolated system is not a constant of the motion, in general, and its variation is bounded, the bounds determined by the thermodynamic entropy, i.e., the equilibrium entropy. We define the nonequilibrium entropy as a convex functional of the set of $n$-particle reduced distribution functions $(n \leqslant N)$ generalizing the Gibbs fine-grained entropy formula. Additionally, as a consequence of our microscopic analysis we find that this nonequilibrium entropy behaves as a free entropic oscillator. In the approach to the equilibrium regime, we find relaxation equations of the Fokker-Planck type, particularly for the one-particle distribution function. (C) 2007 American Institute of Physics. [DOI: 10.1063/1.2800165]
\end{abstract}

\section{INTRODUCTION}

It is a widely recognized fact that a general mathematical theoretical proof of the second law is still lacking. As stated in Ref. 1 and quoted here just for illustration sake "To the best of our knowledge no theoretical mathematical derivation of the second law has been given up until now; instead it has been based on Kelvin's or Clausius's principles of the impossibility of perpetual motion of the second kind, ${ }^{2}$ which are based on experiment." ${ }^{3}$ This lack of definitive theoretical proof has lead to reports on the violation of the second law ${ }^{4}$ or tests over its validity in some particular cases. ${ }^{5,6}$

The first significant contribution to the interpretation of the second law of thermodynamics and the explanation of irreversibility goes back to Boltzmann. Nevertheless, it is known that Boltzmann's contribution was criticized by arguing that this contradicts the predictions based on the microscopic equations of motion. Later, Gibbs and P. Ehrenfest and Ehrenfest worked on this problem by introducing coarse graining. However, those coarse-graining analyses require the introduction of a priori equal probability principles, which are hard to justify on physical grounds as was criticized by Einstein. ${ }^{7}$

In this scenario, our contention is to discern the connection between the microscopic description of an isolated $\mathrm{N}$-body system given through the classical Hamiltonian dynamics and the description at the macroscopic level expressed by the second law.

It is known that for thermodynamic equilibrium the entropy can be given by the Gibbs formula

$$
S_{N}=-k_{B} \operatorname{Tr}(F \ln F),
$$

where $k_{B}$ is the Boltzmann constant and $F$ the full phase-space distribution function which we assume normalized to unity, i.e., $\operatorname{Tr}(F)=1$. However, this expression is not adequate for representing the entropy of nonequilibrium isolated systems for which no bath is present. ${ }^{8}$ The reason is that although in the case of a time-dependent distribution function out of equilibrium, the entropy

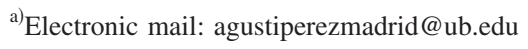


$S$ given through (1) remains constant. This is not difficult to show given that $F$ evolves according to the Liouville equation,

$$
\frac{\partial}{\partial t} F=[H, F]_{p},
$$

where $[\ldots, \ldots]_{P}$ is the Poisson bracket. In fact, by using Eq. (2) the rate of change of the entropy (1) is

$$
\frac{\partial S_{N}}{\partial t}=\operatorname{Tr}\left(\frac{\partial F}{\partial t} \ln F\right)=\operatorname{Tr}\left([H, F]_{p} \ln F\right)=-\operatorname{Tr}\left(F[H, \ln F]_{p}\right)=-\operatorname{Tr}\left([H, F]_{p}\right)=0 .
$$

Therefore here we will generalize Gibbs' statistics to account for the entropy variations in nonequilibrium systems. This constitutes an application of our previous results. ${ }^{9}$ Our starting point is the description of the state of an isolated $N$-body system in terms of the set of $n$-particle reduced distribution functions in the framework of the Bogolyubov-Born-Green-Kirkwood-Yvon (BBGKY) description. ${ }^{10}$ Unlike equilibrium, an overall picture in terms of the full phase-space distribution function does not contain the amount of detail necessary to describe a nonequilibrium system. Nonequilibrium systems manifest a random clusterization which makes their distribution in the phase space unstable, thus there is a continuous process of creation of $n$-particle clusters at the expense of the annihilation of $p$-particle clusters with $n \neq p$. This fact is taken into account in the BBGKY hierarchy, making this an appropriate framework for the description of nonequilibrium systems. In this context, since the collisions become explicit through the collision term in the equations of motion, the $n$-particle reduced distribution functions are not constant of the motion, therefore a way of defining the entropy to embody the approach to equilibrium might be expressed in terms of this set of reduced distributions. This is what we do here: we propose a functional of the set of $n$-particle reduced distribution functions which generalizes the Gibbs entropy as the nonequilibrium entropy of the isolated $N$-body system. We will show that this entropy is not a constant of the motion and reaches its maximum value at equilibrium.

In the next section, we introduce the Hamiltonian dynamics of the $N$-body system and obtain the generalized Liouville equation. In Sec. III, we define the nonequilibrium entropy analyzing its properties. Section IV is devoted to computing the entropy production and to the derivation of the kinetic equation for the one-particle reduced distribution function. In Sec. V we describe the approach to equilibrium. Finally, in Sec. VI, we emphasize our main conclusions.

\section{HAMILTONIAN DYNAMICS}

Let us consider an $N$-body system with a Hamiltonian containing a kinetic energy term plus a two-particle interaction potential,

$$
H=\sum_{j=1}^{N} \frac{\mathbf{p}_{j}^{2}}{2 m}+\frac{1}{2} \sum_{j \neq k=1}^{N} \phi\left(\left|\mathbf{q}_{j}-\mathbf{q}_{k}\right|\right),
$$

with $m$ being the mass of a particle and $\phi\left(\left|\mathbf{q}_{j}-\mathbf{q}_{k}\right|\right) \equiv \phi_{j, k}$ the interaction potential. Moreover, the equations of motion are

$$
\dot{\mathbf{q}}_{i}=\frac{\partial H}{\partial \mathbf{p}_{i}}, \quad \dot{\mathbf{p}}_{i}=-\frac{\partial H}{\partial \mathbf{q}_{i}} .
$$

As said in the Introduction, the statistical description of the system can be performed in terms of the full phase-space distribution function $F\left(x^{N}, t\right)$, where $x^{N}=\left\{x_{1}, \ldots, x_{N}\right\}$ and $x_{j}=\left(\mathbf{q}_{j}, \mathbf{p}_{j}\right)$ or alternatively in terms of the distribution vector ${ }^{11} \mathbf{f}$. Both previous descriptions are completely equivalent, however, the second one is more appropriate for nonequilibrium systems. Here, 


$$
\mathbf{f} \equiv\left\{f_{0}, f_{1}\left(x_{1}\right), f_{2}\left(x^{2}\right), \ldots, f_{N}\left(x^{N}\right)\right\}
$$

is the set of all the $n$-particle reduced distribution functions, with $x^{n}=\left\{x_{1}, \ldots, x_{n}\right\}, n=0, \ldots, N$, and where the $n$-particle reduced distribution functions,

$$
f_{n}=\int F\left(x^{N}, t\right) d x_{n+1} \cdots d x_{N},
$$

are obtained by integrating over the $N-n$ particles with $f_{0}=1$. The dynamics of the reduced distribution vector follows from the Liouville Eq. (2) by integration according to Eq. (7), thus one obtains $^{11-13}$

$$
\frac{\partial}{\partial t} f_{n}=\left[H_{n}, f_{n}\right]_{p}+(N-n) \sum_{j=1}^{n} \int \mathbf{F}_{j, n+1} \frac{\partial}{\partial \mathbf{p}_{j}} f_{n+1} d x_{n+1},
$$

where $\mathbf{F}_{j, n+1}=-\nabla_{j, n+1} \phi_{j, n+1}$ and

$$
H_{n}=\sum_{i=1}^{n} \frac{\mathbf{p}_{i}^{2}}{2 m}+\frac{1}{2} \sum_{i \neq k=1}^{n} \phi_{i, k}
$$

is the $n$-particle Hamiltonian.

In a compact way and in the language of Hilbert spaces we can write Eq. (8) (Refs. 11 and 9)

$$
i \frac{\partial}{\partial t} \mathbf{f}(t)=\mathcal{L} \mathbf{f}(t)
$$

constituting the generalized Liouville equation which succinctly expresses the BBGKY hierarchy of equations. Here, $\mathcal{L}$ is the generalized Liouvillian, a non-Hermitian operator whose diagonal part $\mathcal{P} \mathcal{L}$ is defined through ${ }^{14,11}$

$$
\left\langle n|\mathcal{P} \mathcal{L}| n^{\prime}\right\rangle=i\left[H_{n}, f_{n}\right]_{P} \delta_{n^{\prime}, n}, n>0,
$$

where $|n\rangle$ represents the $n$-particle state. In addition, the nondiagonal part $\mathcal{Q L}$ is given by ${ }^{14,11}$

$$
\left\langle n|\mathcal{Q} \mathcal{L}| n^{\prime}\right\rangle=i\left\{(N-n) \sum_{j=1}^{n} \int \mathbf{F}_{j, n+1} \frac{\partial}{\partial \mathbf{p}_{j}} f_{n+1} d x_{n+1}\right\} \delta_{n^{\prime}, n+1}, \quad n>1 .
$$

Here, $\mathcal{P}$ and $\mathcal{Q}$, its complement with respect to the identity, are projector operators. From its definition through Eq. (11) one can see that $\mathcal{P} \mathcal{L}$ is a $(N+1) \times(N+1)$ diagonal block Hermitian matrix. On the other hand, from Eq. (12) it is possible to infer that $\mathcal{Q L}$ is a non-Hermitian $(N$ $+1) \times(N+1)$ diagonal block matrix with nonzero elements only along the diagonal $(n, n+1)$ with $n>1 .{ }^{11}$ In terms of the projectors just introduced, Eq. (10) can be rewritten,

$$
i \frac{\partial}{\partial t} \mathbf{f}(t)-\mathcal{P} \mathcal{L} \mathbf{f}(t)=\mathcal{Q} \mathcal{L} \mathbf{f}(t) .
$$

Hence, the formal solution of Eq. (13) can be written as an integral equation,

$$
\mathbf{f}(t)=\exp (-i \mathcal{P} \mathcal{L} t) \mathbf{f}(0)+\exp (i \mathcal{P} \mathcal{L} t) \int_{0}^{t} d \tau \exp (-i \mathcal{P} \mathcal{L} \tau)(-i \mathcal{Q L}) \mathbf{f}(\tau)
$$

which can be formally solved to give ${ }^{11}$

$$
\mathbf{f}(t)=\mathcal{U}(t, 0) \mathbf{f}(0),
$$

where the evolution operator $\mathcal{U}(t, 0)$ is given by a perturbative development as 


$$
\mathcal{U}(t, 0)=\sum_{j=0}^{\infty} \int_{0}^{t} d t_{1} \int_{0}^{t_{1}} d t_{2} \int_{0}^{t_{2}} d t_{3} \cdots \int_{0}^{t_{j-1}} d t_{j} \times \mathbf{V}\left(t, t_{1}\right) \cdots \mathbf{V}\left(t_{j-1}, t_{j}\right) \exp \left(-i \mathcal{P} \mathcal{L} t_{j}\right) .
$$

Here, $\mathbf{V}\left(t_{j-1}, t_{j}\right)=\exp \left[i \mathcal{P} \mathcal{L}\left(t_{j-1}-t_{j}\right)\right](-i \mathcal{Q} \mathcal{L})$ are non-Hermitian propagators, $t_{j}<t_{j-1}<\ldots<t_{1}<t_{0}$ $=t$, and the integration proceeds from right to left. Differentiating Eq. (16) one finds

$$
\frac{\partial}{\partial t} \mathcal{U}(t, 0)=-i \mathcal{L U}(t, 0)
$$

the evolution equation for $\mathcal{U}(t, 0)$. If now, we make a time translation and change the origin of the time scale so that the time series begin at time $t$,

$$
\mathcal{U}(0,-t)=\sum_{j=0}^{\infty} \int_{-t}^{0} d t_{1}^{\prime} \int_{-t}^{t_{1}^{\prime}} d t_{2}^{\prime} \int_{-t}^{t_{2}^{\prime}} d t_{3}^{\prime} \cdots \int_{-t}^{t_{j}^{\prime}} d t_{j}^{\prime} \times \mathbf{V}\left(0, t_{1}^{\prime}\right) \cdots \mathbf{V}\left(t_{j-1}^{\prime}, t_{j}^{\prime}\right) \exp \left[-i \mathcal{P} \mathcal{L}\left(t_{j}^{\prime}+t\right)\right]
$$

with $t_{l}^{\prime}=t_{l}-t,(1 \leqslant l \leqslant j)$ and under time reversal

$$
\mathcal{U}(0, t)=\sum_{j=0}^{\infty} \int_{t}^{0} d t_{1} \int_{t}^{t_{1}} d t_{2} \int_{t}^{t_{2}} d t_{3} \cdots \int_{t}^{t_{j-1}} d t_{j} \times \mathbf{V}\left(0, t_{1}\right) \cdots \mathbf{V}\left(t_{j-1}, t_{j}\right) \exp \left[-i \mathcal{P} \mathcal{L}\left(t_{j}-t\right)\right]
$$

Interchanging the integration limits and the integrals we obtain after relabeling the dummy integration variables ${ }^{15}$

$$
\mathcal{U}(0, t)=\sum_{j=0}^{\infty}(-1)^{j} \int_{0}^{t} d t_{1} \int_{0}^{t_{1}} d t_{2} \int_{0}^{t_{2}} d t_{3} \cdots \int_{0}^{t_{j-1}} d t_{j} \times \exp \left(-i \mathcal{P} \mathcal{L} t_{1}\right) \mathbf{U}\left(t_{j}, t_{j-1}\right) \cdots \mathbf{U}\left(t_{1}, t\right)
$$

where now, $\mathbf{U}\left(t_{j}, t_{j-1}\right)=(-i \mathcal{Q} \mathcal{L}) \exp \left[-i \mathcal{P} \mathcal{L}\left(t_{j}-t_{j-1}\right)\right]$. In addition, by differentiating Eq. (20) one gets the evolution equation for $\mathcal{U}(0, t)$,

$$
\frac{\partial}{\partial t} \mathcal{U}(0, t)=\mathcal{U}(0, t) i \mathcal{L}
$$

The propagator $\mathcal{U}(0, t)$ given through Eq. (19) propagates backwards in time from $t$ to 0 , hence this must coincide with the inverse $\mathcal{U}(t, 0)^{-1}$ of $\mathcal{U}(t, 0)$ so that

$$
\mathcal{U}(t, 0)^{-1} \mathbf{f}(t)=\mathcal{U}(0, t) \mathbf{f}(t)=\mathbf{f}(0) .
$$

It can be verified that $\mathcal{U}(0, t)$ is the inverse of $\mathcal{U}(t, 0) .{ }^{15}$ To begin, $\mathcal{U}(0, t) \mathcal{U}(t, 0)=1$ for $t=0$. Now by differentiating and taking into account Eqs. (17) and (21) we reach

$$
\frac{\partial}{\partial t} \mathcal{U}(0, t) \mathcal{U}(t, 0)=0
$$

so $\mathcal{U}(0, t) \mathcal{U}(t, 0)=1$ for all $t$.

Since $\mathcal{P L}$ is Hermitian, all its eigenvalues are real, ${ }^{16}$ which means that $\mathbf{f}(t)$ as given through Eqs. (15) and (16) will have an oscillatory behavior.

\section{NONEQUILIBRIUM ENTROPY}

Here as the nonequilibrium entropy for the $N$-body system we propose $e^{9,14}$ 


$$
S=-k_{B} \operatorname{Tr}\left\{\mathbf{f} \ln \mathbf{f}_{\mathrm{eq}}^{-1} \mathbf{f}\right\}+S_{\mathrm{eq}}=-k_{B} \sum_{n=1}^{N} \int f_{n} \ln \frac{f_{n}}{f_{\text {eq }, n}} d x_{1} \cdots d x_{n}+S_{\mathrm{eq}},
$$

a convex functional of the distribution vector which generalizes the Gibbs formula. In Eq. (24), $S_{\text {eq }}$ is the thermodynamic entropy (i.e., the equilibrium entropy) and $\mathbf{f}_{\mathrm{eq}}$ is the equilibrium distribution vector satisfying $\mathcal{L} \mathbf{f}_{\mathrm{eq}}=0$, the Yvon-Born-Green $(\mathrm{YBG})$ equilibrium hierarchy. ${ }^{11}$ Therefore, $\mathbf{f}_{\mathrm{eq}}$ is an eigenfunction of $\mathcal{L}$ with eigenvalue 0 . Moreover,

$$
\delta S=-k_{B} \operatorname{Tr}\left\{\delta \mathbf{f} \ln \left(\mathbf{f}_{\mathrm{eq}}^{-1} \mathbf{f}\right)\right\}
$$

is zero at equilibrium and

$$
\delta^{2} S=-\frac{1}{2} k_{B} \operatorname{Tr}\left\{\delta \mathbf{f f}_{\mathrm{eq}}^{-1} \delta \mathbf{f}\right\}
$$

is a negative quantity, which shows that $S$ is maximum at equilibrium where its value is $S_{\text {eq. }}$.

Note that the BBGKY scenario describes an interacting mixture of fluids made up of particle clusters in the phase space. Two such fluids differ in the size of the clusters they contain and each fluid contributes its own entropy, the $n$-particle entropy, to the total nonequilibrium entropy of our system. Likewise, the interaction between different fluids leads to the creation of $n$-particle clusters at the expense of the annihilation of $p$-particle clusters with $n \neq p$.

More interestingly here the most important property of the entropy we propose is its direction of change in a natural process. To elucidate this, we must establish the entropy bounds, if any. Hence, let us define the $n$-particle entropies,

$$
S_{n}=-k_{B} \int f_{n} \ln \frac{f_{n}}{f_{\text {eq }, n}} d x_{1} \cdots d x_{n} .
$$

Since the full distribution function $F$ contains more information than $f_{n}$, one might expect that $S_{n} \geqslant S_{N}$. This can be proven from the convexity of the logarithmic function, $\ln x \leqslant x-1$, which can be rewritten ${ }^{17}$

$$
f \ln f-f \ln g \geqslant f-g \quad \text { for } f \geqslant 0, g>0,
$$

where there is strict inequality unless $f=g$. Hence, assuming that $f=F$ and $g=f_{n}$, from Eq. (28) one derives

$$
\int F \ln F d x_{1} \cdots d x_{N} \geqslant \int F \ln f_{n} d x_{1} \cdots d x_{N}=\int f_{n} \ln f_{n} d x_{1} \cdots d x_{n} .
$$

Analogously, it can be proven

$$
\int f_{n} \ln f_{n} d x_{1} \cdots d x_{n} \geqslant \int f_{n} \ln f_{\mathrm{eq}, n} d x_{1} \cdots d x_{n},
$$

which allows us to rewrite Eq. (29),

$$
\int F \ln F d x_{1} \cdots d x_{N} \geqslant \int f_{n} \ln \frac{f_{n}}{f_{\mathrm{eq}, n}} d x_{1} \cdots d x_{n} .
$$

Therefore, from Eqs. (1) and (27),

$$
S_{n} \geqslant S_{N}
$$

and consequently, from Eqs. (24), (27), and (32) one obtains

$$
0 \geqslant S-S_{\text {eq }} \geqslant S_{N} .
$$

In light of this, we find that $S$ is bounded 


$$
0 \geqslant S_{\text {eq }} \geqslant S \geqslant S_{N}+S_{\text {eq }}
$$

This result together with our comments at the end of the previous section leads us to conclude that the nonequilibrium entropy $S$ behaves as a free oscillator with an amplitude of oscillation $\triangle_{S}=$ $-S_{N} / 2$. Hence, there is no possibility of time arrow and a question arises as to how the equilibrium could be reached and more deeply how to reread the second law for isolated systems. We will try to answer these questions in the next section.

To end this section, in view of our previous conclusion we assume the existence of a potential associated with the harmonic entropic oscillator,

$$
\Phi(S)=\frac{1}{2} K_{\text {eff }}(t)\left(S-S^{*}\right)^{2},
$$

with $S^{*}=S_{\text {eq }}+S_{N} / 2$. Therefore, the potential bounds satisfy

$$
\Phi\left(S_{\mathrm{eq}}\right)=\Phi\left(S_{N}+S_{\mathrm{eq}}\right)=\frac{1}{2} K_{\mathrm{eff}}(t)\left(\frac{S_{N}}{2}\right)^{2},
$$

and the effective elastic constant is given through

$$
K_{\text {eff }}(t)=\left|\frac{1}{S} \frac{\partial^{2}}{\partial t^{2}} S\right| .
$$

Consequently,

$$
S-S^{*}=\Delta_{S} \sin \left(\sqrt{K_{\mathrm{eff}}(t)} t+\varphi\right),
$$

where $\varphi$ stands for the initial conditions. This system has a first integral of the motion its "energy" given by ${ }^{18}$

$$
\mathcal{H}(S(t), \dot{S}(t), t)=\frac{1}{2}\left(\frac{\dot{S}(t)}{\sqrt{K_{\mathrm{eff}}(t)}}\right)^{2}+\Phi(S(t)),
$$

which is constant. Moreover, the period of the oscillations $\tau$ satisfies

$$
2 \pi=\tau \sqrt{K_{\text {eff }}(\tau)}
$$

and should be coherent with the recurrence period of the Poincare cycles.

\section{ENTROPY PRODUCTION. THE LAW OF INCREASE OF ENTROPY}

The rate of change of the nonequilibrium entropy or entropy production is obtained by taking the time derivative of Eq. (24), giving

$$
\frac{\partial S}{\partial t}=-k_{B} \operatorname{Tr}\left\{\frac{\partial \mathbf{f}}{\partial t} \ln \left(\mathbf{f}_{\mathrm{eq}}^{-1} \mathbf{f}\right)\right\}=i k_{B} \operatorname{Tr}\left\{\mathcal{L} \mathbf{f} \ln \left(\mathbf{f}_{\mathrm{eq}}^{-1} \mathbf{f}\right)\right\} .
$$

In a more explicit way, after using Eqs. (8) and (10)-(12), Eq. (41) can be rewritten

$$
\frac{\partial S}{\partial t}=-\frac{1}{T} \sum_{n=1}^{N} \sum_{j=1}^{n} \int f_{n} \mathbf{p}_{j}\left(-k_{B} T \frac{\partial}{\partial \mathbf{q}_{j}} \ln f_{\mathrm{eq}, n}+\sum_{j \neq i=1}^{n} \mathbf{F}_{j, i}+(N-n) \mathcal{F}_{j}\right) d x^{n},
$$

where $\mathcal{F}_{j}\left(x^{n}\right)$ is defined through $f_{n}\left(x^{n}\right) \mathcal{F}_{j}\left(x^{n}\right)=\int \mathbf{F}_{j, n+1} f_{n+1} d x_{n+1}$, and $T$ is the kinetic temperature taking into account that the dependence of $f_{\text {eq }, n}$ in the velocities is given through a local Maxwellian. The entropy production given in Eq. (42) vanishes at equilibrium and in any other case it should not be necessarily zero. In addition, because $\mathbf{p}_{j}$ is arbitrary, 


$$
\sum_{j \neq i=1}^{n} \mathbf{F}_{j, i}+(N-n) \mathcal{F}_{j}^{\mathrm{eq}}=k_{B} T \frac{\partial}{\partial \mathbf{q}_{j}} \ln f_{\mathrm{eq}, n}
$$

is sufficient to satisfy the extremum condition $\delta \dot{S} /\left.\delta f_{n}\right|_{\mathrm{eq}}=0$, with $\dot{S}=\partial S / \partial t$. Precisely, Eq. (43) gives rise to the YBG hierarchy. ${ }^{9,19}$ (42) as

On the other hand, by using Eq. (43), we can rewrite the entropy production given through Eq.

$$
\frac{\partial S}{\partial t}=-\frac{1}{T} \sum_{n=1}^{N} \sum_{j=1}^{n}(N-n) \int f_{n} \mathbf{p}_{j}\left(\mathcal{F}_{j}-\mathcal{F}_{j}^{\mathrm{eq}}\right) d x^{n},
$$

which is the starting equation to analyze the relaxation to equilibrium. To this end, as in nonequilibrium thermodynamics ${ }^{20}$ from Eq. (44) we can establish the phenomenological relation

$$
f_{n} \mathbf{p}_{j}=f_{\mathrm{eq}, n} \mathbf{p}_{j}-\sum_{i=1}^{n} \frac{\mathbf{L}_{j, i}}{T}\left(\mathcal{F}_{i}-\mathcal{F}_{i}^{\mathrm{eq}}\right),
$$

where $\mathbf{L}_{j, i}$ is a phenomenological matrix which, in general, might depend on the nonequilibrium thermodynamic force $\left(\mathcal{F}_{i}-\mathcal{F}_{i}^{\mathrm{eq}}\right)$. In terms of the mobility $\mathbf{M}_{j, i}=\mathbf{L}_{j, i} / T f_{n}$ we can rewrite Eq. (45) as

$$
\mathbf{J}_{j}=-\sum_{i=1}^{n} f_{n} \mathbf{M}_{j, i}\left(\mathcal{F}_{i}-\mathcal{F}_{i}^{\mathrm{eq}}\right)
$$

where we have defined the current $\mathbf{J}_{j}=f_{n} \mathbf{p}_{j}-f_{\text {eq, }, n} \mathbf{p}_{j}$, so $\mathbf{J}_{j}$ and $\left(\mathcal{F}_{i}-\mathcal{F}_{i}^{\text {eq }}\right)$ constitute a pair of conjugated current and thermodynamic force, respectively.

Now we are in position to introduce the crucial point that might explain the approach to equilibrium and the link with the macroscopic irreversibility. Thus, near equilibrium, which coincides with the extremum position of the entropic oscillator related to the potential given through Eq. (35), $\mathbf{M}_{j, i} \rightarrow \mathbf{M}_{j, i}^{\mathrm{eq}}$ which in this particular case should be a non-negative constant matrix. Therefore, in this case

$$
\frac{\partial S}{\partial t}=\frac{1}{T} \sum_{n=1}^{N} \sum_{j=1}^{n}(N-n) \int f_{n}\left(\mathcal{F}_{j}-\mathcal{F}_{j}^{\mathrm{eq}}\right) \mathbf{M}_{j, i}^{\mathrm{eq}}\left(\mathcal{F}_{i}-\mathcal{F}_{i}^{\mathrm{eq}}\right) d x^{n} \geqslant 0,
$$

constituting the law of increase of entropy.

\section{RELAXATION EQUATIONS}

In this section we will analyze the relaxation to equilibrium by deriving the relaxation equation for the one-particle reduced distribution function. To obtain such an equation we introduce the inverse mobility matrix $\zeta_{j, i}^{\mathrm{eq}}\left(\sum_{i=1}^{n} \zeta_{j, i}^{\mathrm{eq}} \mathbf{M}_{i, l}^{\mathrm{eq}}=\delta_{j, l}\right)$, the friction matrix which allows us to invert the near equilibrium version of Eq. (46)

$$
f_{n}\left(\mathcal{F}_{i}-\mathcal{F}_{i}^{\mathrm{eq}}\right)=-\sum_{i=1}^{n} \zeta_{i, j}^{\mathrm{eq}} \mathbf{J}_{j}=-\sum_{i=1}^{n} \zeta_{i, j}^{\mathrm{eq}} \mathbf{p}_{j}\left(f_{n}-f_{\mathrm{eq}, n}\right) .
$$

At this point it will be useful to introduce the physical volume of the system $V$ as a scale factor, thus we will redefine the reduced distribution functions. ${ }^{13}$

$$
\hat{f}_{n}=V^{n} f_{n}
$$

Additionally, we must also redefine the forces, writing $\hat{\mathcal{F}}_{i} / V$ and $\hat{\mathcal{F}}_{i}^{\text {eq }} / V$ instead of $\mathcal{F}_{i}$ and $\mathcal{F}_{i}^{\text {eq }}$. Hence, for $n=1$, we obtain from Eq. (8) 


$$
\frac{\partial}{\partial t} \hat{f}_{1}+\mathbf{p} \frac{\partial}{\partial \mathbf{q}} \hat{f}_{1}=\left(\frac{N-1}{V}\right) \frac{\partial}{\partial \mathbf{p}} \hat{f}_{1} \hat{\mathcal{F}}_{1}
$$

Thus, by using Eqs. (43), (48), and (50) we obtain the kinetic equation for $\hat{f}_{1} \equiv f$,

$$
\frac{\partial}{\partial t} f+\mathbf{p} \frac{\partial}{\partial \mathbf{q}} f-k_{B} T\left(\frac{\partial}{\partial \mathbf{q}} \ln f_{\mathrm{eq}}\right) \frac{\partial}{\partial \mathbf{p}} f=-\left(\frac{N-1}{V}\right) \zeta \frac{\partial}{\partial \mathbf{p}} \mathbf{p}\left(f-f_{\mathrm{eq}}\right),
$$

where $\zeta \equiv \zeta_{2,1}^{\mathrm{q}}$. In the thermodynamic limit

$$
\frac{\partial}{\partial t} f+\mathbf{p} \frac{\partial}{\partial \mathbf{q}} f-k_{B} T\left(\frac{\partial}{\partial \mathbf{q}} \ln f_{\mathrm{eq}}\right) \frac{\partial}{\partial \mathbf{p}} f=-\rho \zeta \frac{\partial}{\partial \mathbf{p}} \mathbf{p}\left(f-f_{\mathrm{eq}}\right),
$$

with $\rho=N / V$ being the density. This equation constitutes a generalization of the Bhatnagar-GrossKrook relaxation model. ${ }^{9}$

To illustrate the approach to equilibrium let us write

$$
f(\mathbf{q}, \mathbf{p}, t)=\psi_{\mathbf{q}}(\mathbf{p}, t) \phi(\mathbf{q}, t) .
$$

By introducing the factorization given through Eq. (53) into Eq. (52) after integration in p, we obtain

$$
\frac{\partial \phi}{\partial t}+\frac{\partial}{\partial \mathbf{q}} J_{\phi}=0
$$

where

$$
J_{\phi}=\phi \int \psi_{\mathbf{q}} \mathbf{p} d \mathbf{p}
$$

is the current of the probability density $\phi(\mathbf{q}, t)$ or first moment of the density $\psi_{q}$, which satisfies the equation

$$
\frac{\partial}{\partial t} J_{\phi}+\frac{\partial}{\partial \mathbf{q}} \phi \int \psi_{\mathbf{q}} \mathbf{p} \mathbf{p} d \mathbf{p}+k_{B} T\left(\frac{\partial}{\partial \mathbf{q}} \ln f_{\mathrm{eq}}\right) \phi=\rho \zeta J_{\phi} .
$$

Here, for time $t \gg(\rho \zeta)^{-1}$, Eq. (56) leads to

$$
J_{\phi}=\frac{1}{\rho \zeta} \frac{\partial}{\partial \mathbf{q}} \phi \int \psi_{\mathbf{q}} \mathbf{p} \mathbf{p} d \mathbf{p}+\frac{k_{B} T}{\zeta}\left(\frac{\partial}{\partial \mathbf{q}} \ln f_{\mathrm{eq}}\right) \phi,
$$

which substituted into Eq. (54) and assuming that $\psi_{\mathbf{q}}$ is a local Maxwellian such that $\int \psi_{\mathbf{q}} \mathbf{p p} d \mathbf{p}$ $=-k_{B} T$ gives

$$
\frac{\partial \phi}{\partial t}=-\mathbf{L}_{\mathbf{q}} \phi
$$

where the lineal differential operator $\mathbf{L}_{\mathbf{q}}$ is given through

$$
\mathbf{L}_{\mathbf{q}} \phi=-\frac{k_{B} T}{\rho \zeta} \frac{\partial}{\partial \mathbf{q}}\left(\frac{\partial}{\partial \mathbf{q}} \phi-\phi \frac{\partial}{\partial \mathbf{q}} \ln f_{\mathrm{eq}}\right) .
$$

This equation contains a term $k_{B} T \partial / \partial \mathbf{q} \ln f_{\text {eq }}$ that plays the role of a thermal force usually introduced in polymer dynamics. ${ }^{21}$

In the next section, starting in Eq. (58) and from the properties of $\mathbf{L}_{\mathbf{q}}$ defined through Eq. (59), we will study the approach to equilibrium. 


\section{APPROACH TO EQUILIBRIUM}

The differential operator $\mathbf{L}_{\mathbf{q}}$ introduced in the previous section is a non-Hermitian operator whose Hermitian conjugated is defined through

$$
\mathbf{L}_{\mathbf{q}}^{\dagger} \varphi=-\frac{k_{B} T}{\rho \zeta}\left(\frac{\partial}{\partial \mathbf{q}}+\frac{\partial}{\partial \mathbf{q}} \ln f_{\mathrm{eq}}\right) \frac{\partial}{\partial \mathbf{q}} \varphi .
$$

Therefore, $\mathbf{L}_{\mathbf{q}}$ does not have an orthonormal base. However, we can define right-hand and lefthand eigenfunctions through

$$
\mathbf{L}_{\mathbf{q}} \Omega_{p}(\mathbf{q})=\lambda_{p} \Omega_{p}(\mathbf{q})
$$

and

$$
\mathbf{L}_{\mathbf{q}}^{\dagger} \omega_{p}(\mathbf{q})=\lambda_{p} \omega_{p}(\mathbf{q})
$$

which we chose to be orthonormal, $\int d \mathbf{q} \Omega_{p}(\mathbf{q}) \omega_{r}(\mathbf{q})=\delta_{p, r}$. As has been said in Sec. III, $\phi_{\mathrm{eq}}(\mathbf{q})$ is an eigenfunction with eigenvalue of 0 of the evolution operator $\mathbf{L}_{\mathbf{q}}$, thus it is possible to write

$$
\Omega_{p}(\mathbf{q})=\phi_{\mathrm{eq}}(\mathbf{q}) \omega_{p}(\mathbf{q}),
$$

which can be proven by direct substitution of this relation into Eq. (61) and taking into account Eqs. (59), (60), and (62). So, $\Omega_{0}(\mathbf{q})=\phi_{\text {eq }}(\mathbf{q})$ and $\omega_{0}(\mathbf{q})=1$. On the other hand the eigenvalues different from zero are positive. In fact, by multiplying Eq. (61) by $\omega_{p}(\mathbf{q})$ and integrating, one obtains

$$
\lambda_{p} \int d \mathbf{q} \phi_{\mathrm{eq}} \omega_{p}^{2}=\int d \mathbf{q} \omega_{p} \mathbf{L}_{\mathbf{q}} \phi_{\mathrm{eq}} \omega_{p}=\frac{k_{B} T}{\rho \zeta} \int d \mathbf{q} \phi_{\mathrm{eq}} \frac{\partial}{\partial \mathbf{q}} \omega_{p} \frac{\partial}{\partial \mathbf{q}} \omega_{p} \geqslant 0,
$$

where the right-hand side in the last equality has been obtained by using Eq. (59) and integrating by parts. Also, the positivity of $\zeta$ discussed in the context of Eq. (47) has been taken into account.

Now any distribution $\phi(\mathbf{q}, t)$ can be expanded in terms of the eigenfunctions

$$
\phi(\mathbf{q}, t)=\sum_{p} \alpha_{p}(t) \omega_{p}(\mathbf{q}) \phi_{\mathrm{eq}}(\mathbf{q}),
$$

where taking into account the orthonormality condition

$$
\alpha_{p}(t)=\int d \mathbf{q} \omega_{p}(\mathbf{q}) \phi(\mathbf{q}, t) .
$$

From Eqs. (58), (59), and (65) one obtains the evolution equation for the coefficients of the expansion (65),

$$
\frac{d}{d} \alpha_{p}(t)=-\lambda_{p} \alpha_{p}(t)
$$

which gives

$$
\alpha_{p}(t)=\alpha_{p}(0) \exp \left(-\lambda_{p} t\right) .
$$

Since $\omega_{0}=1$ and $\phi(\mathbf{q}, t)$ should be normalized, $\alpha_{0}=1$. Therefore,

$$
\phi(\mathbf{q}, t)=\phi_{\mathrm{eq}}(\mathbf{q})+\sum_{p} \alpha_{p}(0) \exp \left(-\lambda_{p} t\right) \omega_{p}(\mathbf{q}) \phi_{\mathrm{eq}}(\mathbf{q}),
$$

showing that after a long period of time equilibrium is eventually reached. 


\section{CONCLUSIONS}

We find that the description of an $N$-body isolated system in the framework of the BBGKY hierarchy enables us to prove that the nonequilibrium entropy is not a constant of the motion. We emphasize that the nonequilibrium entropy should be defined as a convex functional of the distribution vector. Our contention is that the adequate functional is the one given in Eq. (24). Moreover, this description reconciles the reversibility of the Hamiltonian dynamics with the approach to equilibrium.

Due to the periodic character of the solution of the microscopic equations given through Eqs. (15) and (16), we realize that the nonequilibrium entropy corresponds to a dynamical system that behaves as a free oscillator, an "entropic oscillator" with well established bounds determined by the equilibrium entropy which is the maximum entropy. We have also manage to construct the Hamiltonian for this entropic oscillator. Hence, the approach to equilibrium occurs when the entropy production is positive, i.e., when the dynamical system is rising through the walls of the elastic potential defined in Eq. (35). In other words, the entropy production is positive when the balance of forces appearing in the integrand of Eq. (42) is opposite to velocity, thus preventing the expansion of the $\mathrm{N}$-body system in the phase space. The natural extension of our theory to study nonisolated systems, i.e., dissipative $N$-body systems, would be to consider a damped entropic oscillator instead a free oscillator. In the case of the damped entropic oscillator, the system collapses in the equilibrium state which is the attractor of the dynamics.

Performing a nonequilibrium thermodynamic analysis, we are able to derive relaxation equations of the Fokker-Planck type, particularly for the one-particle distribution function. Finally, through a spectral analysis we show how these equations describe the approach to equilibrium.

${ }^{1}$ E. G. D. Cohen and D. Mauzurall, J. Stat. Mech.: Theory Exp. 2004, P07006.

${ }^{2}$ N. G. van Kampen, Stochastic Processes in Physics and Chemistry (North-Holland, Amsterdam, 1990).

${ }^{3}$ G. E. Crooks, J. Stat. Phys. 90, 1481 (1998).

${ }^{4}$ G. M. Wang, E. M. Sevick, E. Mittag, D. J. Searles, and D. J. Evans, Phys. Rev. Lett. 89, 050601 (2002).

${ }^{5}$ S. Abe and A. K. Rajagopal, Phys. Rev. Lett. 91, 120601 (2003).

${ }^{6}$ G. W. Ford and R. F. O’Connell, Phys. Rev. Lett. 96, 020402 (2006).

${ }^{7}$ E. G. D. Cohen, Pramana, J. Phys. 64, 635 (2005); Physica A 305, 19 (2002).

${ }^{8}$ N. N. Bogoliubov and N. N. Bogoliubov, Jr., Introduction to Quantum Statistical Mechanics (World Scientific, Singapore, 1982).

${ }^{9}$ A. Pérez-Madrid, J. Stat. Mech.: Theory Exp. 2006, P09015.

${ }^{10}$ N. N. Bogoliubov, Lectures on Quantum Statistics (Gordon and Breach, New York, 1967), Vol. 1.

${ }^{11}$ R. Balescu, Equilibrium and Non-Equilibrium Statistical Mechanics (Wiley-Interscience, New York, 1975); see also R. Balescu, Statistical Dynamics. Matter out of Equilibrium (Imperial College Press, London, 1997).

${ }^{12}$ H. J. Kreuzer, Nonequilibrium Thermodynamics and its Statistical Foundations (Clarendon, Oxford, 1981).

${ }^{13}$ L. C. Woods, The Thermodynamics of Fluid Systems (Clarendon, Oxford, 1975).

${ }^{14}$ A. Pérez-Madrid, Physica A 339, 339 (2004).

${ }^{15}$ D. J. Evans and G. P. Morriss, Statistical Mechanics of Nonequilibrium Liquids (Academic, London, 1990).

${ }^{16}$ R. C. Tolman, The Principles of Statistical Mechanics (Dover, New York, 1980).

${ }^{17}$ H. Grad, Commun. Pure Appl. Math. 14, 323 (1961).

${ }^{18}$ M. V. Bartuccelli and G. Gentile, Phys. Lett. A 307, 274 (2003).

${ }^{19}$ T. L. Hill, Statistical Mechanics. Principles and Selected Applications (Dover, New York, 1987).

${ }^{20}$ S. R. de Groot and P. Mazur, Non-Equilibrium Thermodynamics (Dover, New York, 1984).

${ }^{21}$ R. B. Bird, C. F. Curtiss, R. C. Armstrong, and O. Hassager, Dynamics of Polymeric Liquids, Kinetic Theory, Vol. 2, 2nd ed. (Wiley, New York, 1987). 